\title{
AN HI LINE SURVEY OF POLAR RING GALAXIES
}

\author{
W. VAN DRIEL \\ Unité Scientifique Nançay, Observatoire de Paris, France \\ M. ARNABOLDI \\ Osservatorio Astronomico di Capodimonte, Naples, Italy \\ F. COMBES \\ DEMIRM, Observatoire de Paris, France \\ AND

\section{L.S. SPARKE} \\ Astronomy Dept., University of Wisconsin-Madison, U.S.A.
}

We have observed a total of 74 polar ring galaxies (PRGs), PRG candidates and related objects at 3 radio observatories: Green Bank (Richter et al. 1994, $A J$, 107, 1), Effelsberg (Huchtmeier 1997, $A \& A, 319,401)$ and Nançay (van Driel et al. 1997, $A \mathscr{G} A S$, in press; van Driel et al. 1998, $A \mathcal{E} A$, in prep.). Our main aim is to identify systems suitable for $\mathrm{H}$ I synthesis mapping, crucial for an understanding of their dynamical state. Most objects were selected using one or more of the following 3 criteria: known redshift $(<8000 \mathrm{~km} / \mathrm{s})$, or blue magnitude brighter than $15.5 \mathrm{mag}$, or kinematically confirmed PRG or good PRG candidate. A total of 62 objects were detected, 39 of which with more than one telescope.

A large range in gas-richness is found among the objects observed. The kinematically confirmed PRGs are all gas-rich, with an average $M_{\mathrm{HI}} / L_{\mathrm{B}}$ ratio of $1.1 M_{\odot} / L_{\odot, \mathrm{B}}$ (typical for gas-rich late-type galaxies), while the PRG candidates and related objects show a much larger range; some are as gas-rich as confirmed PRGs, while a lot of them have as little $\mathrm{H}$ I as detected normal S0 galaxies (about $0.15 M_{\odot} / L_{\odot, \mathrm{B}}$ ) - radio synthesis imaging of PRGs shows the $\mathrm{H}$ I to be associated with the polar ring and not with the equatorial S0 disk. The Tully-Fisher (TF) relation, made without correcting profile widths for inclination (a correct procedure for true PRGs with their edge-on rings), shows the largest deviations from the average relation for non-confirmed PRG candidates and related objects. 